\title{
Quantitative Analysis of Uniaxial Molecular Orientation in Langmuir- Blodgett Films by Infrared Reflection Spectroscopy
}

\section{$\operatorname{AUTHOR}(\mathrm{S})$ :}

Hasegawa, T.; Takeda, S.; Kawaguchi, A.; Umemura, J.

\section{CITATION:}

Hasegawa, T. ... [et al]. Quantitative Analysis of Uniaxial Molecular Orientation in LangmuirBlodgett Films by Infrared Reflection Spectroscopy. Langmuir 1995, 11(4): 1236-1243

\section{ISSUE DATE:}

1995-04

URL:

http://hdl.handle.net/2433/217712

\section{RIGHT:}

This document is the Accepted Manuscript version of a Published Work that appeared in final form in 'Langmuir', copyright (c) American Chemical Society after peer review and technical editing by the publisher. To access the final edited and published work see http://dx.doi.org/10.1021/la00004a032. 


\section{Quantitative Analysis of Uniaxial Molecular Orientation in Langmuir-Blodgett Films by Infrared Reflection Spectroscopy}

T. Hasegawa, ${ }^{\dagger}$ S. Takeda, ${ }^{+}$A. Kawaguchi ${ }^{\mathrm{I}}$ and J. Umemura* Institute for Chemical Research, Kyoto University

Uji, Kyoto-Fu, 611 Japan

*Author to whom correspondence should be sent.

†Present address : Kobe Pharmaceutical University, Motoyama-kitamachi, Higashinada-ku, Kobe 658, Japan.

"Present address : Research Center, Asahi Glass Co., Ltd., 1150 Hazawacho, Kanagawa-ku, Yokohama 221, Japan.

IPresent address : Department of Applied Chemistry, Faculty of Science and Engineering, Ritsumeikan University, Kusatsu, Siga 525, Japan 


\section{Abstract}

In analyzing optical properties of multilayered LB films with the uniaxial anisotropy around the surface normal, a new method for calculating infrared reflection-absorbances was developed, by extending Drude's anisotropic calculation theory for two-phase system to Hansen's optical theory for thin isotropic multilayers. With this method, infrared external reflection spectra of a 9-monolayer LB film of cadmium stearate prepared on a gallium arsenide substrate were analyzed to obtain molecular orientations. The result was in fair agreement with that obtained by X-ray diffractometry. Reflection-absorbance spectra of the same LB film on a silver-evaporated slide glass at various temperatures were also analyzed by the same method and the orientation angle of each molecular group was quantitatively obtained, clarifying the process of disordering with the increase of temperature. Further, the dependence of the degree of disordering on the monolayer location in LB films were discussed in light of the isotope substitution experiment.

\section{Introduction}

In recent years, Langmuir-Blodgett (LB) films have been investigated in various fields for extending the possibilities of many kinds of organic ultra-thin membrane devices. ${ }^{1,2}$ LB films are also used for fundamental physical studies because their thickness can be controlled at will on a nanometer scale. In order to fabricate the high-quality LB film, it is desirable to know the molecular orientation in the LB film in situ and non-destructively. For this purpose, the infrared (IR) spectroscopic technique is very suitable. In particular, a recent Fourier transform (FT) IR spectrophotometer has fairly good sensitivity and signal-to-noise ratio, so that ultra-thin LB film even at a monolayer level can 
be measured. In practice, transmission measurements in combination with reflection-absorption (RA) measurements ${ }^{3}$ are often used for analyzing molecular orientations in LB films since the electric vector in the transmission measurements of LB films on IR-transparent materials is parallel to the film surface, while that in the RA measurements of LB films on metal surfaces is perpendicular to it. For instance, Umemura et al. ${ }^{4}$ quantitatively estimated the molecular orientation angle in the 9-monolayer LB film of cadmium stearate by this method. Ozaki et al. also applied this method to other functionalized LB films. ${ }^{5,6}$ As another way in evaluating molecular orientation, Yarwood et al. proposed the way of attenuated total reflection (ATR) spectra in combination with RA spectra. ${ }^{79}$ They investigated the nature and extent of molecular ordering in alternate-layer LB films of 4- $n$-heptadecylamido-4'-nitrostilbene/stearic acid and a functionalized diarylalkyne (JT11) with this method. ${ }^{9}$ However, these methods have disadvantage in that molecular orientations of LB films in transmission (or ATR) and RA measurements are sometimes different because of the difference in substrates used for both measurements. On the other hand, the ordinary infrared external reflection (ER) measurements of LB films on non-metallic substrates have some advantage in that it gives negative or positive reflection-absorbances, depending upon the direction of the transition moment, the angle of incidence, and the polarization of incident beam. ${ }^{10 \sim 12}$ For example, the molecular orientation of Langmuir film (spread monolayer on water) was studied by the FT-IR ER technique. ${ }^{13,14}$ Here, the reflection-absorbance $A$ is defined by the equation : $A=\log \left(R_{0} / R\right)$, where $R_{0}$ and $R$ are reflectances without and with film sample on substrate, respectively. On the other hand, Mielczarski and Yoon $^{15}$ studied the molecular orientation in spontaneously adsorbed ethyl xanthate $\left(\mathrm{C}_{2} \mathrm{H}_{5} \mathrm{OCS}_{2}^{-}\right.$ion) on a semiconductor, cuprous sulfide (chalcocite), from aqueous solutions by the infrared ER technique. In this study, however, 
the observed spectra were discussed by using Hansen's approximate formulae ${ }^{16}$ for calculating theoretical reflection-absorbance values of the three-phase (air-thin film-substrate) system. These approximate equations were derived from Hansen's exact ones ${ }^{16}$ by expanding them in terms of $\alpha d^{17}: \alpha$ is the absorption coefficient given by $\alpha \equiv 4 \pi k / \lambda$ where $k$ is the extinction coefficient of the film, $\lambda$ the wavelength in vacuo, and $d$ the thickness of the film. Thus, these formulae, along with similar approximation formulae derived by McIntyre, ${ }^{18,}$ ${ }^{19}$ do not hold when the film is too thick or when the film has strongly absorbing structural groups. What is more, these are based on the optically isotropic approximation for the complex refractive indices. In evaluating the orientation angle of an oriented molecule rigorously, optical anisotropy should be considered. Recently, Parikh and Allara ${ }^{11}$ have applied the rigorous $4 \times 4$ transfer matrix theory developed by Yeh for multilayered anisotropic films. ${ }^{20}$ With the aid of complex refractive indices obtained by the Kramers-Kronig transformation, they succeeded to analyze the structure of highly organized thin films on gold, carbon, and silica.

In this study, much easier and convenient theoretical method was developed for the uniaxially oriented LB film, by anisotropically expanding Hansen's isotropic optical theory for multilayered system. ${ }^{16}$ Though this expansion could be done very easily, obtained new equations can be applied to the analysis of the molecular orientation in thin LB films on any kinds of substrates.

\section{Experimental}

Pure stearic acid used in this study was the same as that reported previously. ${ }^{21}$ Distilled water was made by a modified Mitamura Riken Model PLS-DFR automatic lab still consisting of a reverse osmosis module, an ion-exchange column, and a double distiller. Langmuir films of cadmium stearate were 
prepared by spreading a $1.0 \mathrm{mg} / \mathrm{mL}$ benzene solution of stearic acid on water containing $3 \times 10^{-4} \mathrm{M} \mathrm{CdCl}_{2}$ and buffered with $3 \times 10^{-4} \mathrm{M} \mathrm{NaHCO}_{3}$ to $\mathrm{pH} 6.2$. All the reagents were of highly pure (>98\%) or spectroscopic grade (benzene). After waiting $10 \mathrm{~min}$ to allow the solvent to be fully evaporated, the Langmuir film was compressed at the compression velocity of $19.5 \mathrm{~cm}^{2} / \mathrm{min}$ up to the surface pressure of $30 \mathrm{mN} / \mathrm{m}$, and then transferred by the conventional LB method $^{22}$ onto finely polished gallium arsenide circular wafers with the (100) crystalline plane which were produced in Sumitomo Electric Industries, Ltd. The gallium arsenide wafers had been doped with silicon at carrier concentration of $1.8 \times 10^{26} \mathrm{~cm}^{-3}$. The wafers had been cleaned by successive ultrasonication in ethanol, acetone, and dichloromethane for 10 minutes each at $20{ }^{\circ} \mathrm{C}$ in advance. Dipping and withdrawing speeds of the wafers were $5 \mathrm{~mm} / \mathrm{min}$, and the transfer ratio was $0.97 \pm 0.05$ throughout the experiments. For RA measurements, 100 $\mathrm{nm}$ thick silver-evaporated slide glass was used as a substrate. The silver evaporation was done at a speed of $2 \AA / \mathrm{sec}$ under vacuum of approximately $6 \times 10^{-7}$ Torr.

FT-IR spectra in ER measurements and in RA measurements of isotopically substituted LB films were recorded on a Nicolet Model 710 FT-IR spectrophotometer, and those in other RA and transmission measurements were recorded on a Nicolet Model $6000 \mathrm{C}$ spectrophotometer. All measurements were performed with a resolution of $4 \mathrm{~cm}^{-1}$, using MCT detectors. For reflection measurements, a Harrick Model RMA-1DG/VRA versatile reflection attachment with retro-mirror and focus transfer accessory was used. The $p$ - and $s$-polarized beams were obtained through a Hitachi $\mathrm{Au} / \mathrm{AgBr}$ wire-grid polarizer. The degree of polarization of the polarizer was $98 \%$ at $3000 \mathrm{~cm}^{-1}$. With decreasing the wavenumber, the degree of polarization was improved. For example, it was above $99 \%$ at the wavenumber below $2000 \mathrm{~cm}^{-1}$. In order to improve the 
degree of polarization, we tried to use two polarizers in parallel with each other. In this condition, however, the through-put was lowered, so that the signal-to-noise ratio $(\mathrm{S} / \mathrm{N})$ was degraded. Then, we were obliged to use a single polarizer, although the degree of polarization would effect the spectroscopic property near the Brewster angle. For ER measurements, the angle of incidence $\theta$ was $50^{\circ}$ or $30^{\circ}$ in the $p$ - or $s$-polarized beams, respectively, and $\theta$ was $85^{\circ}$ for RA measurements. The $p$-and $s$-polarized infrared spectra of the same substrates without LB films were measured as references. The number of interferogram accumulations was 5000, 3000, and 500 for ER, RA, and transmission measurements, respectively.

In elevating the temperature of $\mathrm{LB}$ films for RA measurements, the same method was used as that reported previously. ${ }^{23}$ For the transmission measurements of $\mathrm{KBr}$ pellets, a Hitachi IRH-3 vertical heating cell equipped with two $\mathrm{NaCl}$ windows was used. The temperature of the sample was determined by a copper-constantan thermocouple, whose reference was fixed at $0{ }^{\circ} \mathrm{C}$ by a Komatsu-Electronics ZC-114 zero compensator.

The spacing of the LB film was determined by $X$-ray diffractometry using a Shimazu VD-1 diffractometer operated at $30 \mathrm{kV}-30 \mathrm{~mA}$. The divergence slit was $0.24 \mathrm{~mm}$ and the receiving slit was $0.20 \mathrm{~mm}$.

\section{Results and Discussion}

\section{(A) Theoretical section}

First, we need to calculate reflection and transmission coefficients as well as the electric field intensities in any layer for $p$ - and $s$-polarized beams in obtaining theoretical reflection-absorbances. In order to prevent too complex calculation, the uniaxial optical property which is often the case in the LB film 
was assumed. In this condition, two optical constants are inevitable as anisotropic optical constants. These are $\tilde{n}_{j o}$ and $\tilde{n}_{j e}$ whose principal axis are parallel to the surface parallel ( $x$ - or $y$-direction in Fig. 1 ) and the surface normal ( $z$-direction in Fig. 1) of the LB film, respectively. Here, the subscript $j$ refers to the location number of the stratified layer, and $o$ or $e$ stands for ordinary $(s-$ polarization) or extraordinary ( $p$-polarization), respectively. The wave symbols $(\sim)$ are attached to all complex values in this paper. The meaning of introduction of anisotropic optical constants in calculation is as follows. In general, if there is a uniaxial anisotropy in a crystal, the incident natural ray is divided into two rays with $s$ - and $p$-polarizations. In these rays, the $s$-polarized ray refracts according to Snell's law, while the $p$-polarized ray does not follow the law. The isotropic theory is applicable to the $s$-polarized ray, since Snell's law holds well in an isotropic medium. Hansen's theory which is based on the optical isotropic assumption is widely known in calculating optical properties in stratified media. In his theory, the term, $\tilde{\xi}\left(=\left(\tilde{n}_{j}^{2}-n_{1}^{2} \sin ^{2} \theta_{1}\right)^{1 / 2}\right)$, is defined to be $\tilde{n}_{j} \cos \tilde{\theta}_{j}$. This definition is based on Snell's law and for the s-polarized ray in a uniaxial anisotropic system, $\tilde{\xi}=\left(\tilde{n}_{j o}^{2}-n_{1}^{2} \sin ^{2} \theta_{1}\right)^{1 / 2}=\tilde{n}_{j o} \cos \widetilde{\theta}_{j}$. In $p$-polarization measurements, the propagation direction is different from that in the $s$ polarization. ${ }^{24}$ Since this is the intrinsic difference in the present phenomena, the definition of $\tilde{\xi}$ should be modified for the $p$-polarization. For this purpose, the following equation by Drude ${ }^{25,26}$ (eq 1) was referred. Drude's equations are those for calculating reflection coefficient for 2-layer systems comprising an isotropic layer and a uniaxial anisotropic layer. From the isotropic layer, a ray is incident at various angles of incidence. The problem is to extend this for any number of stratified layer systems. According to Drude, the reflection coefficient at the boundary between 1 st and 2 nd layer, $\tilde{r}_{12}$, for $p$-polarized beam can be written by 


$$
\tilde{r}_{12}=\frac{\tilde{n}_{2 o} \tilde{n}_{2 e} \cos \theta_{1}-\tilde{n}_{1 o}\left(\tilde{n}_{2 e}{ }^{2}-\tilde{n}_{1 o}{ }^{2} \sin ^{2} \theta_{1}\right)^{1 / 2}}{\tilde{n}_{2 o} \tilde{n}_{2 e} \cos \theta_{1}+\tilde{n}_{1 o}\left(\tilde{n}_{2 e}{ }^{2}-\tilde{n}_{1 o}{ }^{2} \sin ^{2} \theta_{1}\right)^{1 / 2}} .
$$

First, this equation was transformed into the next one :

$$
\begin{aligned}
\tilde{r}_{12}= & \frac{\frac{1}{\widetilde{n}_{1 o}} \cos \theta_{1}-\frac{1}{\tilde{n}_{2 o} \widetilde{n}_{2 e}}\left(\tilde{n}_{2 e}^{2}-\tilde{n}_{1 o}^{2} \sin ^{2} \theta_{1}\right)^{1 / 2}}{\frac{1}{\widetilde{n}_{1 o}} \cos \theta_{1}+\frac{1}{\widetilde{n}_{2 o} \widetilde{n}_{2 e}}\left(\tilde{n}_{2 e}{ }^{2}-\tilde{n}_{1 o}{ }^{2} \sin ^{2} \theta_{1}\right)^{1 / 2}} \\
& =\frac{A-B}{A+B}
\end{aligned}
$$

where

$$
\begin{aligned}
& A=\frac{1}{\widetilde{n}_{1 o}} \cos \theta_{1} \\
& B=\frac{1}{\widetilde{n}_{2 o} \widetilde{n}_{2 e}}\left(\widetilde{n}_{2 e}^{2}-\widetilde{n}_{1 o}^{2} \sin ^{2} \theta_{1}\right)^{1 / 2} .
\end{aligned}
$$

Next, for investigating the relation between Drude's equation and Hansen's equation, the same term, $\tilde{r}_{12}$, can be written in Hansen's way ${ }^{16}$ as eq 5

$$
\tilde{r}_{12}=\frac{\left(\widetilde{m}_{11}+\widetilde{m}_{12} \widetilde{q}_{2}\right) \widetilde{q}_{1}-\left(\widetilde{m}_{21}+\widetilde{m}_{22} \widetilde{q}_{2}\right)}{\left(\widetilde{m}_{11}+\widetilde{m}_{12} \widetilde{q}_{2}\right) \widetilde{q}_{1}+\left(\widetilde{m}_{21}+\widetilde{m}_{22} \widetilde{q}_{2}\right)}
$$

where $\tilde{q}_{j}=\cos \tilde{\theta}_{j} / \tilde{n}_{j o}($ for $j=1,2)$, and since a 2-layer system is considered, the following relation can be referred.

$$
\left[\begin{array}{ll}
\widetilde{m}_{11} & \widetilde{m}_{12} \\
\widetilde{m}_{21} & \widetilde{m}_{22}
\end{array}\right]=\left[\begin{array}{cc}
\cos \tilde{\beta}_{2} & -\frac{i}{\widetilde{q}_{2}} \sin \tilde{\beta}_{2} \\
-\frac{i}{\widetilde{q}_{2}} \sin \widetilde{\beta}_{2} & \cos \widetilde{\beta}_{2}
\end{array}\right]
$$

Thus, eq 5 was quite simplified as eq 7 by eq 6 .

$$
\tilde{r}_{12}=\frac{\tilde{q}_{1}-\tilde{q}_{2}}{\widetilde{q}_{1}+\widetilde{q}_{2}}
$$

Dimensions of $A, B, \widetilde{q}_{1}$, and $\widetilde{q}_{2}$ are all the same (reciprocal dimension of refractive index). Then, eqs 2 and 7 was compared to obtain principles of transformation 
from isotropic equation to anisotropic one.

$$
\begin{aligned}
& \widetilde{q}_{1}=\frac{\cos \theta_{1}}{\widetilde{n}_{1 o}} \rightarrow A=\frac{\cos \theta_{1}}{\widetilde{n}_{1 o}} \\
& \widetilde{q}_{2}=\frac{\cos \widetilde{\theta}_{2}}{\widetilde{n}_{2 o}} \rightarrow B=\frac{1}{\widetilde{n}_{2 o} \widetilde{n}_{2 e}}\left(\widetilde{n}_{2 e}{ }^{2}-\widetilde{n}_{1 o}{ }^{2} \sin ^{2} \theta_{1}\right)^{1 / 2}
\end{aligned}
$$

The relation (8) has no deep meaning, since there is no change in the transformation. This is because the 1st layer was assumed to be isotropic in this model. ${ }^{25,26}$ The key in the transformation is the relation (9). This relation can be simplified as follows :

$$
\cos \tilde{\theta}_{2} \rightarrow \frac{\tilde{\xi}_{2}}{\tilde{n}_{2 e}}
$$

In this way, the principle of transformation was obtained. This can be generalized to be :

$$
\cos \widetilde{\theta}_{j} \rightarrow \frac{\tilde{\xi}_{j}}{\widetilde{n}_{j e}} .
$$

Here, $\tilde{\xi}_{j}$ is defined to be $\tilde{\xi}_{j}=\left(\tilde{n}_{j e}^{2}-\tilde{n}_{1 o}^{2} \sin ^{2} \theta_{1}\right)^{1 / 2}$. The mathematical value of this notation is changed from Hansen's one, since Snell's law does not hold for $p$-polarized beam in anisotropic system as mentioned above. Then, other substitutions for parameters in Hansen's equation follow. From the relation (11), $\tilde{q}_{j}$ can be written by

$$
\tilde{q}_{j}=\frac{\cos \tilde{\theta}_{j}}{\widetilde{n}_{j o}} \rightarrow \frac{\widetilde{\xi}_{j}}{\widetilde{n}_{j o} \widetilde{n}_{j e}} .
$$

Further, the relation (11) was applied to $\tilde{\beta}_{\dot{j}}$

$$
\tilde{\beta}_{j}=\frac{2 \pi}{\lambda} h_{j} \tilde{n}_{j o} \cos \tilde{\theta}_{j} \rightarrow \frac{2 \pi}{\lambda} h_{j} \tilde{n}_{j o} \widetilde{\xi}_{\tilde{n}_{j e}}
$$


where $h_{j}$ is the thickness of the thin film of $j$ th layer, and $\lambda$ the wavelength of the incident beam. After replacing $\tilde{q}_{j}$ by relation 12 , the rest equations in the paper of Hansen are unnecessary to change. Those equations are described below.

$$
\begin{aligned}
& \widetilde{M}_{j}=\left[\begin{array}{cc}
\cos \tilde{\beta}_{j} & \frac{-i}{\widetilde{q}_{j}} \sin \tilde{\beta}_{j} \\
-i \tilde{q}_{j} \sin \tilde{\beta}_{j} & \cos \widetilde{\beta}_{j}
\end{array}\right] \\
& \tilde{N}_{k}(z)=\left[\begin{array}{cc}
\cos \left(\frac{2 \pi}{\lambda} \tilde{\xi}_{k}\left(z-z_{k-1}\right)\right) & \frac{i}{\widetilde{q}_{k}} \sin \left(\frac{2 \pi}{\lambda} \tilde{\xi}_{k}\left(z-z_{k-1}\right)\right) \\
i \tilde{q}_{k} \sin \left(\frac{2 \pi}{\lambda} \tilde{\xi}_{k}\left(z-z_{k-1}\right)\right) & \cos \left(\frac{2 \pi}{\lambda} \tilde{\xi}_{k}\left(z-z_{k-1}\right)\right)
\end{array}\right] \\
& \tilde{r}_{\mathrm{p}}=\frac{\left(\widetilde{m}_{11}+\widetilde{m}_{12} \widetilde{q}_{N}\right) \widetilde{q}_{1}-\left(\widetilde{m}_{21}+\widetilde{m}_{22} \widetilde{q}_{N}\right)}{\left(\widetilde{m}_{11}+\widetilde{m}_{12} \widetilde{q}_{N}\right) \widetilde{q}_{1}+\left(\widetilde{m}_{21}+\widetilde{m}_{22} \widetilde{q}_{N}\right)} \\
& \tilde{t}_{H \mathrm{p}}=\frac{2 \widetilde{q}_{1}}{\left(\widetilde{m}_{11}+\widetilde{m}_{12} \widetilde{q}_{N}\right) \widetilde{q}_{1}+\left(\widetilde{m}_{21}+\widetilde{m}_{22} \widetilde{q}_{N}\right)} \\
& \tilde{t}_{E \mathrm{p}}=\frac{\tilde{n}_{1 o}}{\tilde{n}_{N o}} \tilde{t}_{H \mathrm{p}} \\
& R_{\mathrm{p}}=\left|\tilde{r}_{\mathrm{p}}\right|^{2}, \quad \delta_{\mathrm{p}}^{r}=\arg \tilde{r}_{\mathrm{p}} \\
& T_{\mathrm{p}}=\frac{\operatorname{Re}\left(\widetilde{q}_{N}\right)}{\operatorname{Re}\left(\widetilde{q}_{1}\right)}\left|\tilde{t}_{H \mathrm{p}}\right|^{2}, \quad \delta_{\mathrm{p}}{ }^{t}=\arg \tilde{t}_{E_{\mathrm{p}}}
\end{aligned}
$$

where notations of all variables, except for $\tilde{n}_{j o}$ and $\tilde{n}_{j e}$, used here are along those in Hansen's paper. ${ }^{16} \widetilde{M}_{j}$ is the complex characteristic matrix, and $\widetilde{N}_{k(z)}$ is the reciprocal of $\widetilde{M}_{j}$. The reflection and transmission coefficients for $p$-polarized electric vector are represented by $\tilde{r}_{\mathrm{p}}$ and $\tilde{t}_{E \mathrm{p}}$. The phase changes for reflection and transmission at any interfaces are indicated by $\delta_{\mathrm{p}}{ }^{r}$ and $\delta_{\mathrm{p}}{ }^{t}$, respectively. $R_{\mathrm{p}}$ and $T_{\mathrm{p}}$ are reflectance and transmittance, respectively. Here, the permeability was always assumed to be unity. Axiomatically, these equations are reduced to 
be that of Hansen's isotropic equations if $\tilde{n}_{j o}=\tilde{n}_{j e}$. With these equations, $R_{\mathrm{P}}+T_{\mathrm{P}}=1$ holds well whenever each layer is non-absorbing. In the case of $s$-polarization, substituting $\tilde{n}_{j o}$ for $\tilde{n}_{j e}$ in eq 11,12 and 13 , and putting $\tilde{p}_{j}=\tilde{n}_{j o} \cos \tilde{\theta}_{j}$, the conventional Hansen's equations are valid. With the above equations, absorbances and electric field intensities in anisotropic multilayered system can be calculated at will. Actual results for infrared reflection measurements will be seen in the following examples.

\section{(B) ER measurements of LB film of cadmium stearate on GaAs}

We have reported the molecular orientation in LB films of cadmium stearate on gallium arsenide wafer analyzed by infrared ER technique with Hansen's approximate formulae where only the extinction coefficient $k$ in LB films were treated anisotropically, under the assumption that refractive index $n$ was isotropic. ${ }^{12}$ In the present treatment, refractive index $n$ can also be anisotropic. The ER spectra of the 9-monolayer LB film on gallium arsenide are shown in Figure 2. The angle of incidence $\theta_{1}$ in $p$-polarization measurements was chosen to be $50^{\circ}$ because intensity of reflection-absorbance contains experimental error to a certain extent when $\theta_{1}$ is near the Brewster angle ( $73^{\circ}$ in this case) ${ }^{12}$ In the case of $s$-polarization measurements, $\theta_{1}$ was chosen as $30^{\circ}$. In $p$-polarization measurements, bands due to the antisymmetric and symmetric $\mathrm{CH}_{2}$ stretching vibrations (2917 and $2850 \mathrm{~cm}^{-1}$, respectively), antisymmetric $\mathrm{COO}^{-}$stretching vibration $\left(1543 \mathrm{~cm}^{-1}\right.$ ) and $\mathrm{CH}_{2}$ scissoring vibration (1462 and $1472 \mathrm{~cm}^{-1}$ ) give negative peaks, while the symmetric $\mathrm{COO}^{-}$stretching band $\left(1433 \mathrm{~cm}^{-1}\right)$ and band progression due to the $\mathrm{CH}_{2}$ wagging modes $\left(1400 \sim 1200 \mathrm{~cm}^{-1}\right)$ give positive peaks. In $s$-polarization measurements, all bands show negative peaks. The symmetric $\mathrm{COO}^{-}$stretching band and the band progression are very weak in absolute intensity. By the calculation method mentioned above, the 
reflection-absorbances in $p$-polarization measurements for the transition moments perfectly oriented parallel or perpendicular to the film surface, $A_{p x}{ }^{\circ}$ and $A_{p z}{ }^{\circ}$, as well as $A_{s x}{ }^{\circ}$ and $A_{s z}{ }^{\circ}$ in $s$-polarization measurements, can be obtained. In the calculation, the refractive indices $n_{o}=1.48$ and $n_{e}=1.56$ (the real part of $\tilde{n}_{o}$ and $\tilde{n}_{e}$ ) obtained by ellipsometry ${ }^{27}$ for a behenic acid monolayer in the solid condensed state where the hydrocarbon chain axis is oriented normal to the surface were borrowed, since there is no reliable infrared data and the change in $n$ of saturated alkanes is very slight throughout near- and mid-IR region. ${ }^{28,29}$ The extinction coefficient of the symmetric $\mathrm{CH}_{2}$ stretching vibration $\left(\nu_{\mathrm{s}}\left(\mathrm{CH}_{2}\right)\right)$ of the stearyl group at $2850 \mathrm{~cm}^{-1}$ in the bulk crystal $\left(k_{b u l k}\right)$ is known to be 0.2 at room temperature. ${ }^{30}$ Thus, $k_{o}\left(=k_{x}=k_{y}\right)$ and $k_{e}\left(=k_{z}\right)$ are determined to be $3 k_{b u l k} / 2=0.3$ and $3 k_{b u l k}=0.6$, respectively. ${ }^{12}$ Accordingly, $n_{1}=1.0, \tilde{n}_{20}=1.48+0.3 \mathrm{i}, \tilde{n}_{2 e}=1.56$, $n_{3}=3.28,{ }^{31} v=2850 \mathrm{~cm}^{-1}, \theta=50^{\circ}$ and $h=22.5 \mathrm{~nm}$ were adopted for calculation of $A_{p x}{ }^{\circ}$ and $A_{s x}{ }^{\circ}$, while the values of $\tilde{n}_{20}=1.48$ and $\tilde{n}_{2 e}=1.56+0.6 \mathrm{i}$ were substituted instead in calculating $A_{p z}{ }^{\circ}$ and $A_{s z}{ }^{\circ}$. The calculated results are shown by solid lines in Figure 3. The dotted line in the same figure represents the results obtained by isotropic Hansen's approximate formulae in which $n_{2}$ (refractive index of LB film) was $1.48\left(n_{2 x}=n_{2 y}=n_{2 z}=1.48\right)$. By considering the anisotropy of refractive index, the values of $A_{p z}{ }^{\circ}$ have significantly changed from the isotropic case. The values of $A_{p x}{ }^{\circ}$ and $A_{s x}{ }^{\circ}$, were not changed at all, since $n_{20}$ stays constant at $1.48 . A_{s z}{ }^{\circ}$ is always zero, since the electric vector is always normal to the transition moment.

Next, the orientation angles were calculated as follows. Here, an ellipsoid of extinction coefficient is considered, because of the optical anisotropy of the uniaxial crystal. This ellipsoid can be represented by the following equations:

$$
\begin{aligned}
& k_{2 o}=k_{x}=k_{y}=\frac{3}{2} k_{\text {bulk }} \sin ^{2} \phi \\
& k_{2 e}=k_{z}=3 k_{\text {bulk }} \cos ^{2} \phi
\end{aligned}
$$


where $\phi$ is the orientation angle. With eq 11 20, the reflection-absorbances in $p$-polarization measurements, $A_{p}$, were calculated for various orientation angles, $\phi$. By comparing the theoretical value $A_{p}$ with the observed value $A_{o b s}$, the most appropriate orientation angle was determined.

In this manner, the orientation angle of each transition dipole moment was calculated by the theoretical values only in $p$-polarization measurements throughout this study. Since the extinction coefficient, $k_{b u l k}$, of $v_{s}\left(\mathrm{CH}_{2}\right)$ was 0.2 , the rest extinction coefficients at $25^{\circ} \mathrm{C}$ were determined by transmission spectra in the $\mathrm{KBr}$ pellet to be $0.264,0.251$, and 0.110 for $v_{\mathrm{a}}\left(\mathrm{CH}_{2}\right), v_{\mathrm{a}}\left(\mathrm{COO}^{-}\right)$, and $v_{s}\left(\mathrm{COO}^{-}\right)$, respectively. The calculated results for various orientation angle were summarized in Table 1. From the values of the orientation angles, $\alpha$ and $\beta$, of the transition dipole moment of $v_{\mathrm{a}}\left(\mathrm{CH}_{2}\right)$ and $v_{\mathrm{s}}\left(\mathrm{CH}_{2}\right)$, the tilt angle $\gamma$ of the molecular axis of the hydrocarbon chain of cadmium stearate from the surface normal is determined to be $13.8^{\circ}$, since these three directions are mutually orthogonal, ${ }^{4}$

$$
\cos ^{2} \alpha+\cos ^{2} \beta+\cos ^{2} \gamma=1 .
$$

Repeated experiments gave similar values. This value is somewhat bigger than that $\left(7^{\circ}\right)$ measured by transmission and RA spectra. ${ }^{4}$ Thus, $\mathrm{X}$-ray diffraction measurements were performed for the present LB film. The result is shown in Figure 4. The four peaks are apparently seen at $2 \theta=3.58,5.33,7.12$ and $8.89^{\circ}$ corresponding to the 2nd, 3rd, 4th and 5th order diffractions, respectively. Since the wavelength of the $\mathrm{CuK}-\alpha$ line is $1.54 \AA$, the bilayer long spacing is determined to be $4.95 \mathrm{~nm}$ as the mean value. On the other hand, the spacing of 21 -monolayer LB film of cadmium stearate on a slide glass was $5.03 \mathrm{~nm} .^{32,33}$ Thus, it is suggested that the structure of the LB film made on a gallium arsenide substrate is slightly different from that on the slide glass. According to Sugi et al. ${ }^{34}$ the bilayer long spacing, $d$ in nm, of cadmium salt of $n$-fatty acid 
changes with the number of carbon atoms $n$ as :

$$
d=0.53+s n
$$

where $s$ is a constant. In cadmium stearate, $n$ is 18 , so that $s$ is determined to be $0.246 \mathrm{~nm}$. Since the typical value of the distance between alternate carbon atoms in alkyl chain is $0.254 \mathrm{~nm},{ }^{35}$ the chain tilting angle $\gamma$ of the LB film on gallium arsenide is calculated to be $\cos ^{-1}(0.246 / 0.254)=14.4^{\circ}$ in good agreement with that obtained by infrared ER measurements within experimental error. Since, as deduced from the $\mathrm{S} / \mathrm{N}$ ratio in Fig. 2, these angles may well have experimental errors of $\pm 1^{\circ}$. Thus, it can be concluded that the observed orientation angles by ER measurements are reliable. In the same way, the orientation angle of the dipole moment of $v_{s}\left(\mathrm{COO}^{-}\right)$was $15.1^{\circ}$ which is a little smaller than that $\left(18^{\circ}\right)$ of the LB films prepared on a slide glass. ${ }^{4}$ In Table 1 , only the absolute band intensity of $v_{\mathrm{a}}\left(\mathrm{COO}^{-}\right)$is much stronger than that expected from theoretical value $A_{p x}{ }^{\circ}(-0.0080)$, so that the orientation angle was not calculated. The exact reason is not clear for the moment. However, three possible reasons may be proposed: (1) electrons are transferred from the substrate to the cadmium cation since the gallium arsenide wafer was the silicon-doped n-type semiconductor, (2) the dipole interaction in the thin LB film made on the gallium arsenide wafer is different from that in the bulk crystal, or (3) The shape of the most intense $v_{\mathrm{a}}\left(\mathrm{COO}^{-}\right)$band in ERS is highly distorted due to its anomalous dispersion in refractive index, with an appreciably different frequency from that in the transmission spectrum. ${ }^{26}$ Possibilities (1) and (2) could cause the extinction coefficient of $v_{a}\left(\mathrm{COO}^{-}\right)$in the $\mathrm{LB}$ film on a gallium arsenide wafer to be different from that of the bulk crystal in $\mathrm{KBr}$ pellet. The most feasible possibility (3) may well cause the inaccuracy of the observed absolute intensity.

Using above equations, the other simulations such as the dependence of 
reflection-absorbance on film thickness can be made. The result of reflection-absorbance in $p$-polarization measurements for the symmetric $\mathrm{CH}_{2}$ stretching band, $A_{p x}^{\circ}$, is shown in Figure 5. Adopted optical constants were the same as those mentioned above. For comparison, the result of $A_{p x}$ obtained by Hansen's approximate formula ${ }^{16}$ with the same optical constants, except for $\tilde{n}_{2}=1.48+0.3 \mathrm{i}$, is shown in the same figure. If the film thickness is thinner than $50 \mathrm{~nm}$, there is no appreciable difference between more exact and approximate values. Above $50 \mathrm{~nm}$, however, the difference starts to appear and becomes significant above $100 \mathrm{~nm}$. Thus, when a film to be examine is thick, the more exact treatment is necessary. The dependence of reflection-absorbance on extinction coefficient of LB film $\left(k_{2}\right)$ was also examined, but there were only small differences between the two values in the range from $k_{2}=0$ to $k_{2}=2.0$.

\section{(C) RA study of thermal stability in LB films of cadmium stearate}

As previously reported, ${ }^{23}$ the thermal stability of 9-monolayer LB film of cadmium stearate was studied qualitatively by infrared RA spectra using the surface selection rule ${ }^{36}$ which is characteristic of infrared RA measurements.

In this study, our new theoretical method was applied to the same RA spectra to monitor the change in the molecular orientation angle quantitatively with elevating temperature of the LB film. The spectra of the RA measurements are shown in Figure 6. Using eq 11 20, dependences of reflection-absorbance of $v_{s}\left(\mathrm{CH}_{2}\right)$ on the angle of incidence in RA measurements at $30^{\circ} \mathrm{C}$ were calculated. The adopted optical constants are the same as those in the previous section (B), except for $\tilde{n}_{3}$ (silver) $=0.62+25.1 \mathrm{i}, 2.20+47.2 \mathrm{i}$, and $2.56+50.7 \mathrm{i}$ at ca. 2900,1543 , and $1433 \mathrm{~cm}^{-1}$, respectively. ${ }^{4}$ The results are shown in Figure 7 where both $A_{p x}{ }^{\circ}, A_{p z}{ }^{\circ}$ and $A_{s x}{ }^{\circ}$ in RA measurements were drawn. By now, in discussing RA measurements, only $A_{p z}{ }^{\circ}$ has been taken into account and $A_{p x}{ }^{\circ}$ has generally been 
ignored by the reason that $A_{p x}{ }^{\circ}$ is negligibly weak. ${ }^{37}$ However, $A_{p x}{ }^{\circ}$ calculated in this study, especially at around the angle of incidence of $85^{\circ}$, is regarded to be significant. Consequently, $k_{2 o}$ was also taken into account as well as $k_{2 e}$ in calculating $A_{p}$. Besides, $k_{b u l k}$ of the $\mathrm{KBr}$ pellet was measured as a function of temperature. The obtained values of $k_{b u l k}$ at various temperature are shown in Figure 8. Then, with eq 21 and 22 , the orientation angles at each temperature were calculated. ${ }^{38}$ In the case of $v_{\mathrm{s}}\left(\mathrm{CH}_{2}\right)$ at $30^{\circ} \mathrm{C}$, the orientation angle $\phi$ is $85.4^{\circ}$ for $A_{o b s}=0.0023$, while the orientation angle of the same band is $83.2^{\circ}$ when putting $k_{20}=0\left(A_{p x}^{\circ}=0\right)$. On the other hand, the orientation angle for $v_{\mathrm{a}}\left(\mathrm{CH}_{2}\right)$ at $30^{\circ} \mathrm{C}$ is $83.2^{\circ}$. When putting $k_{2 o}=0$, it was calculated to be $81.6^{\circ}$. From these results, the tilt angle of hydrocarbon chain $\gamma$ is calculated to be $8.2^{\circ}$, while it is calculated to be $10.8^{\circ}$ when putting $k_{2 o}=0$. It should be noted that the value $8.2^{\circ}$ is apparently smaller than the value $10.8^{\circ}$, as much as $24 \%$. Then, it is found that this uniaxial anisotropic evaluation method is especially significant when the orientation angle is relatively small. The obtained result, $8.2^{\circ}$, is very close to that $\left(7^{\circ}\right)$ obtained by the transmission and RA method. ${ }^{4}$ Orientation angles for various bands and for the hydrocarbon chain axis calculated at various temperatures are were summarized in Figure 9. With increasing temperatures, the orientation angle of the hydrocarbon chain axis $\gamma$ slightly increased below $90{ }^{\circ} \mathrm{C}$, but it drastically increases in the range $90 \sim 110{ }^{\circ} \mathrm{C}$, and is saturated above $120^{\circ} \mathrm{C}$. In the case of $v_{a}\left(\mathrm{COO}^{-}\right)$and $v_{s}\left(\mathrm{COO}^{-}\right)$, on the other hand, the values of the orientation angles themselves are different from that of the hydrocarbon chain, but their temperature dependences are fully corporated with each other. This result is principally consistent with that reported by Naselli et al. ${ }^{39}$ With the increase in temperature above $90^{\circ} \mathrm{C}$, these orientation angles were changed toward the so-called magic angle, $54.7^{\circ}$ which corresponds to the completely disordered state, indicating that the present analysis gives very 
reasonable values.

\section{(D) RA study of an effect of monolayer location on thermal stability}

The above experiments were carried out about homogeneous LB films. However, our new method can be applied to orientation evaluation of any kinds of multilayered heterogeneous LB films. In this section, therefore, the method was applied to the study of the effect of monolayer location on thermal stability. ${ }^{40}$ In order to examine the effect of monolayer location on the order-disorder phase transition, three kinds of 9-monolayer LB films of cadmium stearate- $\mathrm{d}_{35}$ (represented as 'D') in which the 1st, 5th and 9th layer from the substrate was substituted by undeuterated stearates (represented as ' $\mathrm{H}$ ') were prepared. The schematic models of the three, denoted by $1 \mathrm{H} 8 \mathrm{D}, 4 \mathrm{D} 1 \mathrm{H} 4 \mathrm{D}$ and $8 \mathrm{D} 1 \mathrm{H}$, are illustrated in Figure 10. The observed RA spectra are summarized and reproduced in Figure 11. The band intensities in the $\mathrm{CH}_{2}$ stretching vibration region are very weak because only a monolayer of cadmium stearate is contributed to this region. However, appreciably good signal-to-noise ratios are found for such a thin layer. All of the films display similar spectral changes in this figure. Then, the evaluation method was applied. At $2850 \mathrm{~cm}^{-1}, k_{\text {bulk }}=0.2$ and $k_{\text {buk }}=0$ were adopted for the $\mathrm{H}$ and $\mathrm{D}$ layer, respectively. The thickness of each monolayer was $2.5 \mathrm{~nm}$. The rest optical constants were the same as those used in the former section $(\mathrm{C})$. The results for the orientation angles are shown in Figure 12. All the transition temperatures are around $100{ }^{\circ} \mathrm{C}$. At low temperature, there is no large difference in $\gamma$ among different monolayer locations. At 122 ${ }^{\circ} \mathrm{C}$, however, the orientation angle of the $v_{\mathrm{s}}\left(\mathrm{CH}_{2}\right)$ becomes $59.8^{\circ}, 62.7^{\circ}$, and $66.1^{\circ}$ and the values of $\gamma$ are $50.4^{\circ}, 44.3^{\circ}$ and $38.2^{\circ}$, respectively, when the undeuterated stearate monolayer is located in the 1st, 5th, and 9th layer, respectively. These results suggest that the farther the location becomes from 
the substrate, the lower the chain disorder is, or the higher the thermal stability is. This may be because the internal pressure originated from the attraction forces among molecules in LB film decreases as the location of the layer becomes far from the substrate. ${ }^{40,41}$ In this way, the new evaluation method could clearly specify the change of the molecular orientation with the monolayer location, much better than the previous semi-quantitative method..$^{40,41}$

\section{Conclusion}

In this study, Hansen's optical theory for multilayered thin films were developed with the aid of Drude's theoretical speculation to build up a new method for calculating the infrared reflection-absorbances for uniaxial anisotropic LB films. By using this method, the molecular orientation angles in LB films on the gallium arsenide wafer and on the silver-evaporated slide glass were obtained. From these analyses, it was found that the developed calculation method is especially useful for sensitive RA measurements, where precise orientation angles were obtained by considering the value of $A_{p x}^{\circ}$. Finally, additional informations for thermal stability of the LB films of cadmium stearate were clarified with this method.

\section{Acknowledgment}

The authors thank professor emeritus Tohru Takenaka of this institute for his warm encouragement during this work. They are indebted to Dr. Koji Tada of Sumitomo Electric Industries, Ltd. for his kind gift of gallium arsenide wafers used in the present study. This work was supported by the Grant-in-Aid for Scientific Research 03640406 from the Ministry of Education, Science and Culture, Japan, to whom the authors' thanks are due. 


\section{References}

(1) Sugi, M.; Sakai, K.; Saito, M.; Kawabata, Y.; Iijima, S.Thin Solid Films 1985, 69, 132.

(2) Kamata, T.; Umemura, J.; Takenaka, T.; Koizumi, N. J. Phys. Chem. 1991, 95,4092 .

(3) Greenler, R. G. J. Chem. Phys. 1966, 44, 310.

(4) Umemura, J.; Kamata, T.; Kawai, T.; Takenaka, T. J. Phys. Chem. 1990, 94, 62.

(5) Enomoto, S.; Ozaki, Y.; Kuramoto, N. Langmuir 1993, 9, 3219.

(6) Terashita, S.; Nakatsu, K.; Ozaki, Y.; Mochida, T.; Araki, T.; Iriyama, K. Langmuir 1992, 8, 3051.

(7) Song, Y. P.; Petty, M. C.; Yarwood, J.; Feast, W. J.; Tsibouklis, J.; Mukherjee, S. Langmuir 1992, 8, 257.

(8) Lukes, P. J.; Petty, M. C.; Yarwood, J. Langmuir 1992, 8, 3043.

(9) Song, Y. P.; Petty, M. C.; Yarwood, J.; Feast, W. J.; Tsibouklis, J.; Mukherjee, S. Langmuir 1992, 8, 262.

(10) Allara, D. L. ; Nuzzo, R. G. Langmuir 1985, 1, 52.

(11) Parikh, A. N.; Allara, D. L.; J. Chem. Phys. 1992, 96, 927.

(12) Hasegawa, T.; Umemura, J.; Takenaka, T. J. Phys. Chem. 1993, 97, 9009.

(13) Dluhy, R. A. J. Phys. Chem. 1986, 90, 1373.

(14) Gericke, A.; Michailov, A. V.; Hühnerfuss, H. Vib. Spectrosc. 1993, 4, 335 .

(15) Mielczarski, J. A.; Yoon, R. H. J. Phys. Chem. 1989, 93, 2034.

(16) Hansen, W. N. J. Opt. Soc. Am. 1968, 58, 380.

(17) Hansen, W. N. Symp. Faraday Soc. No.4 (The Faraday Soc., London, 1970) p.27.

(18) McIntyre, J. D. E.; Aspnes, D. E. Surface Sci. 1971, 24, 417. 
(19) McIntyre, J. D. E. Symp. Faraday Soc. 1970, 4, 61.

(20) Yeh, P.; J. Opt. Soc. Am. 1979, 69, 742.

(21) Kimura, F.; Umemura, J.; Takenaka, T. Langmuir 1986, 2, 96.

(22) Blodgett, K. B. J. Am. Chem. Soc. 1934, 56, 495.

(23) Hasegawa, T.; Kamata, T.; Umemura, J.; Takenaka, T. Chem. Lett. 1990, 1990, 1543.

(24) Hasegawa, T.; Umemura, J.; Takenaka, T. Appl. Spectrosc. 1993, 47, 338.

(25) Drude, P. Ann. Physik U. Chem. N. F. 1889, 32, 584.

(26) Ishino, Y.; Ishida, H. Langmuir, 1988, 4, 1341.

(27) Paudler, M.; Ruths, J.; Riegler, H. Langmuir 1992, 8, 184.

(28) Goplen, T. G.; Cameron, D. G.; Jones, R. N. Appl. Spectrosc. 1980, 34, 657.

(29) Pearl, J.; Ngoh, M.; Ospina, M.; Khanna, R. J. Geophys. Res. 1992, 96 , 17477.

(30) Popenoe, D. D.; Stole, S. M.; Porter, M. D. Appl. Spectrosc. 1992, 46, 79.

(31) Kudo, K. Kiso Bussei Zuhyo (Tables for fundamental solid state physics) (Kyoritsu Shuppan, Tokyo, 1972) p.454.

(32) Kamata, T.; Umemura, J.; Takenaka, T. Chem. Lett. 1988, 1988, 1231.

(33) Matsuda, A.; Sugi, M.; Fukui, T.; Iijima, S.; Miyahara, M.; Otsubo, Y. J. Appl. Phys. 1977, 48, 771.

(34) Sugi, M; Fukui, T; Iijima, S; Iriyama, K. Bull. Electrotech. Lab. 1979, 43, 825.

(35) Abrahamsson, S; Sydow, E. von Acta Cryst. 1954, 7, 591.

(36) for example : Chollet, P. A.; Messier, J.; Rosilio, C. J. Chem. Phys. 1976, $64,1042$.

(37) Allara, D. L.; Naselli, C. Langmuir 1985, 1, 52.

(38) It is possible that the use of $k_{b u l k}$ is not suitable enough for the analysis of 
LB film, since the melting behavior can be different between the bulk and film. ${ }^{39,40}$ Thus, it is the second best choice. However, at least, it may be suitable enough at low temperatures where the LB film is ordered and at above $120^{\circ} \mathrm{C}$ where it is fully disordered.

(39) Naselli, C.; Rabolt, J. F.; Swalen, J. D. J. Chem. Phys. 1985, 82, 2136.

(40) Umemura, J.; Takeda, S.; Hasegawa, T.; Kamata, T.; Takenaka, T.;

Spectrosimica Acta. 1994, 50A, 1563.

(41) Umemura, J.; Takeda, S.; Hasegawa, T.; Takenaka, T. J. Mol. Struct. 1993, $297,57$. 
Table.1 Orientaion angles $\phi$ of the transition moments obtained using extinction coefficients in bulk crystal $\left(k_{\text {bulk }}\right)$ and observed reflection-absorbances $\left(A_{\text {obs }}\right.$ ) of typical bands in 9-monolayer LB film of Cd stearate on GaAs.

\begin{tabular}{ccccc}
\hline Band & $\mathrm{cm}^{-1}$ & $k_{\text {bulk }}$ & $A_{\text {obs }}$ & $\phi /$ degree \\
\hline$v_{\mathrm{a}}\left(\mathrm{CH}_{2}\right)$ & 2917 & 0.264 & -0.0125 & 78.3 \\
$v_{\mathrm{s}}\left(\mathrm{CH}_{2}\right)$ & 2850 & 0.200 & -0.0102 & 83.0 \\
$v_{\mathrm{a}}\left(\mathrm{COO}^{-}\right)$ & 1543 & 0.251 & -0.0119 & - \\
$v_{\mathrm{s}}\left(\mathrm{COO}^{-}\right)$ & 1433 & 0.110 & 0.00598 & 15.1 \\
\hline
\end{tabular}




\section{Figure captions}

Figure 1 Schematic model for heterogeneous stratified and parallel layered uniaxial system. $\tilde{n}_{o}$ and $\tilde{n}_{e}$ denote ordinary and extra ordinary complex refractive indices, respectively.

Figure 2 Infrared external reflection spectra of 9-monolayer LB film of $\mathrm{Cd}$ stearate prepared on GaAs wafer measured by $s$-polarized (upper) and $p$-polarized (bottom) beams at $30^{\circ}$ and $50^{\circ}$, respectively.

Figure 3 Calculated anisotropic $\left(n_{x}=n_{y}=1.48, n_{z}=1.56\right)$ reflection-absorbances for $v_{s}\left(\mathrm{CH}_{2}\right)$ band at $2850 \mathrm{~cm}^{-1}$ of 9-monolayer LB film of Cd stearate prepared on GaAs as a function of the angle of incidence. The reflection-absorbance components for the transition moment oriented perfectly parallel $\left(A_{p x}{ }^{\circ}\right.$ and $\left.A_{s x}{ }^{\circ}\right)$ or perpendicular $\left(A_{p z}{ }^{\circ}\right.$ and $\left.A_{s z}{ }^{\circ}\right)$ to the film surface are shown. Dashed lines show isotropic $\left(n_{x}=n_{y}=n_{z}=1.48\right)$ results. For other optical constants, see the text.

Figure $4 \mathrm{X}$-ray diffraction pattern of 9-monolayer LB film of Cd stearate on GaAs wafer.

Figure 5 Calculated reflection-absorbance for $v_{s}\left(\mathrm{CH}_{2}\right)$ at $2850 \mathrm{~cm}^{-1}$ of $\mathrm{LB}$ films of $\mathrm{Cd}$ stearate on GaAs wafer as a function of film thickness. Exact results are shown by solid line, and approximate results are shown by dotted line.

Figure 6 Reflection-absorbance spectra of 9-monolayer LB film of Cd stearate on Ag-evaporated slide glass at various temperatures.

Figure 7 Calculated anisotropic reflection-absorbances for $v_{s}\left(\mathrm{CH}_{2}\right)$ band at $2850 \mathrm{~cm}^{-1}$ of 9-monolayer LB film of Cd stearate prepared on Agevaporated slide glass as a function of the angle of incidence. The reflection-absorbance components for the transition moment oriented 
perfectly parallel $\left(A_{p x}{ }^{\circ}\right.$ and $\left.A_{s x}{ }^{\circ}\right)$ or perpendicular $\left(A_{p z}{ }^{\circ}\right.$ and $\left.A_{s z}{ }^{\circ}\right)$ to the film surface are shown. Insert is an enlarged figure in which dotted line shows $A_{s x}{ }^{\circ}$. For the optical constants, see the text.

Figure 8 The extinction coefficients $\left(k_{b u l k}\right)$ of $v_{\mathrm{a}}\left(\mathrm{CH}_{2}\right)(\square), v_{\mathrm{s}}\left(\mathrm{CH}_{2}\right)(\bullet)$, $v_{\mathrm{a}}\left(\mathrm{COO}^{-}\right)(\boldsymbol{\Delta})$ and $v_{\mathrm{s}}\left(\mathrm{COO}^{-}\right)(\Delta)$ bands in bulk crystal of $\mathrm{Cd}$ stearate in $\mathrm{KBr}$ pellet.

Figure 9 Temperature dependence of orientation angles in 9-monolayer LB film of Cd stearate. $(\square): v_{\mathrm{a}}\left(\mathrm{CH}_{2}\right),(\bullet): v_{\mathrm{s}}\left(\mathrm{CH}_{2}\right),(\Delta): v_{\mathrm{a}}\left(\mathrm{COO}^{-}\right)$, $(\boldsymbol{\Delta}): v_{\mathrm{s}}\left(\mathrm{COO}^{-}\right)$and $(\boldsymbol{Q}): \gamma$.

Figure 10 Schematic model for 9-monolayer LB film of Cd stearate- $\mathrm{d}_{35}$ in which 1 st, 5 th, or 9th layer is substituted by undeuterated Cd stearate.

Figure 11 Infrared reflection-absorbance spectra of (a) 1H8D, (b) 4D1H4D, and (c) $8 \mathrm{D} 1 \mathrm{H}$ in the $\mathrm{C}-\mathrm{H}$ stretching vibration region at various temperatures.

Figure 12 Temperature dependence of orientation angles in LB films of (a) $1 \mathrm{H} 8 \mathrm{D}$, (b) $4 \mathrm{D} 1 \mathrm{H} 4 \mathrm{D}$, and (c) $8 \mathrm{D} 1 \mathrm{H}$ in the $\mathrm{C}-\mathrm{H}$ stretching vibration region. $(\square): v_{\mathrm{a}}\left(\mathrm{CH}_{2}\right),(\bullet): v_{\mathrm{s}}\left(\mathrm{CH}_{2}\right)$ and $(\square): \gamma$. 


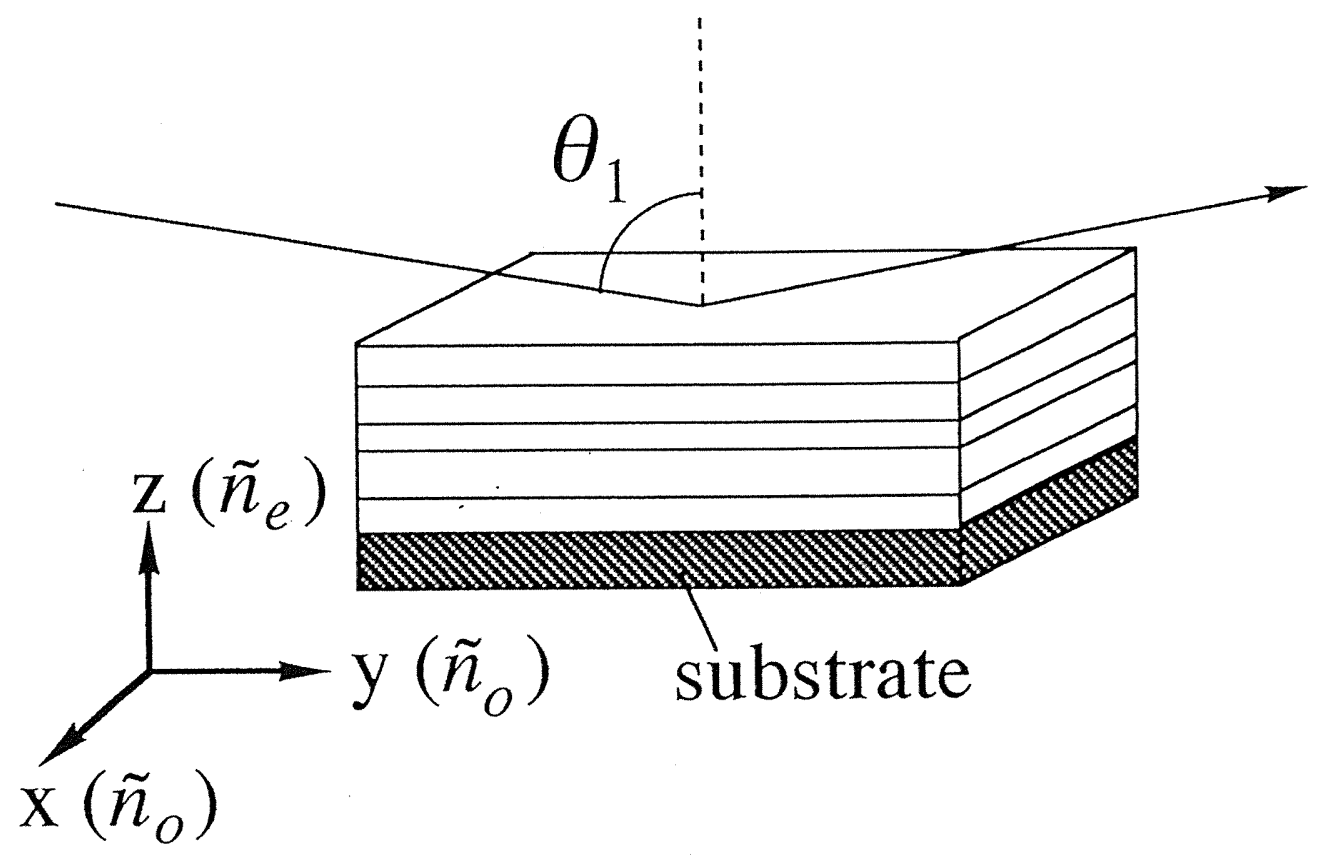

Fig. 1. T. Hasegawa, S. Takeda, A. Kawaguchi and J. Umemura 




Fig. 2. T. Hasegawa, S. Takeda, A. Kawaguchi and J. Umemura 


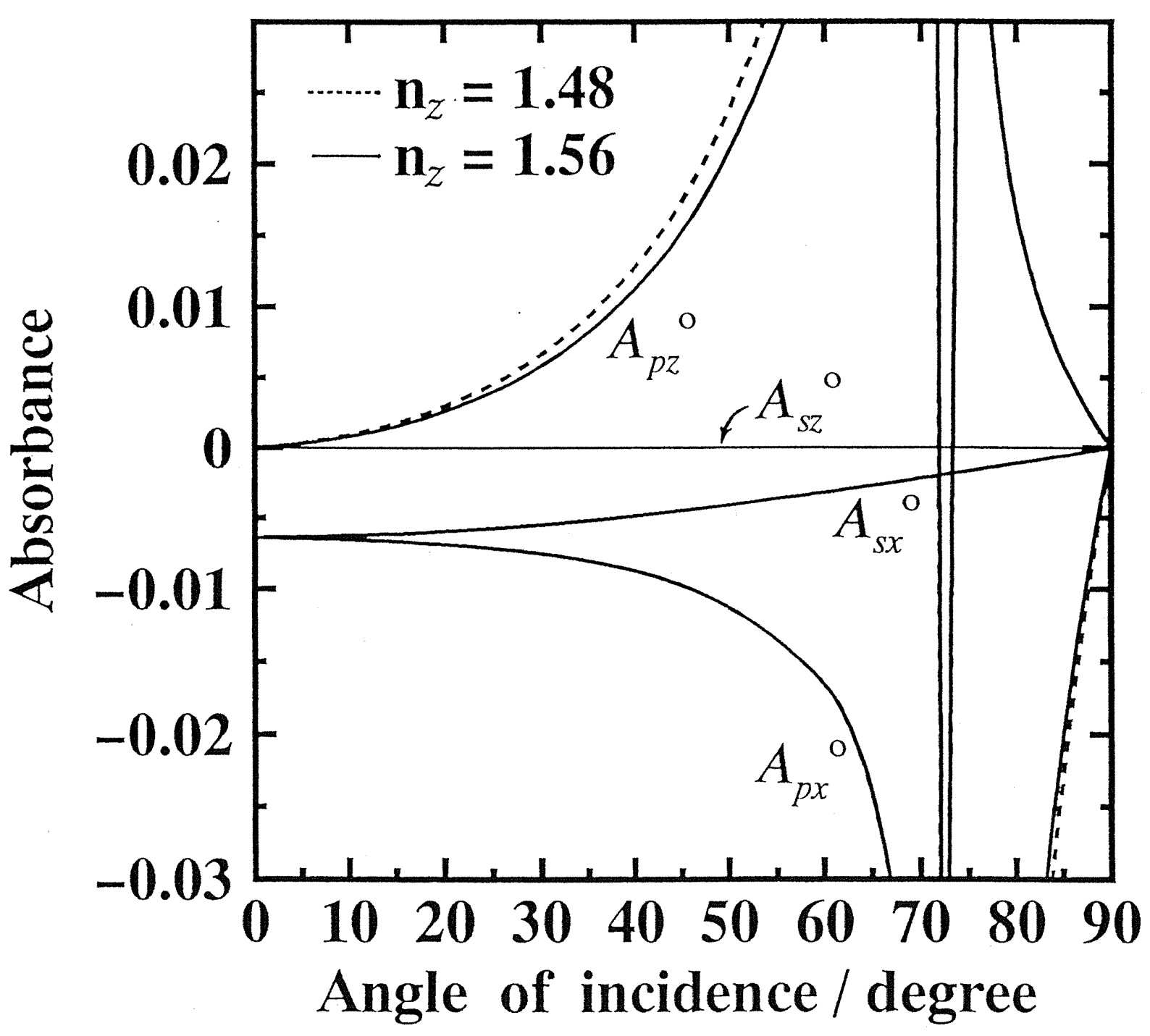

Fig. 3. T. Hasegawa, S. Takeda, A. Kawaguchi and J. Umemura 




Fig. 4. T. Hasegawa, S. Takeda, A. Kawaguchi and J. Umemura 


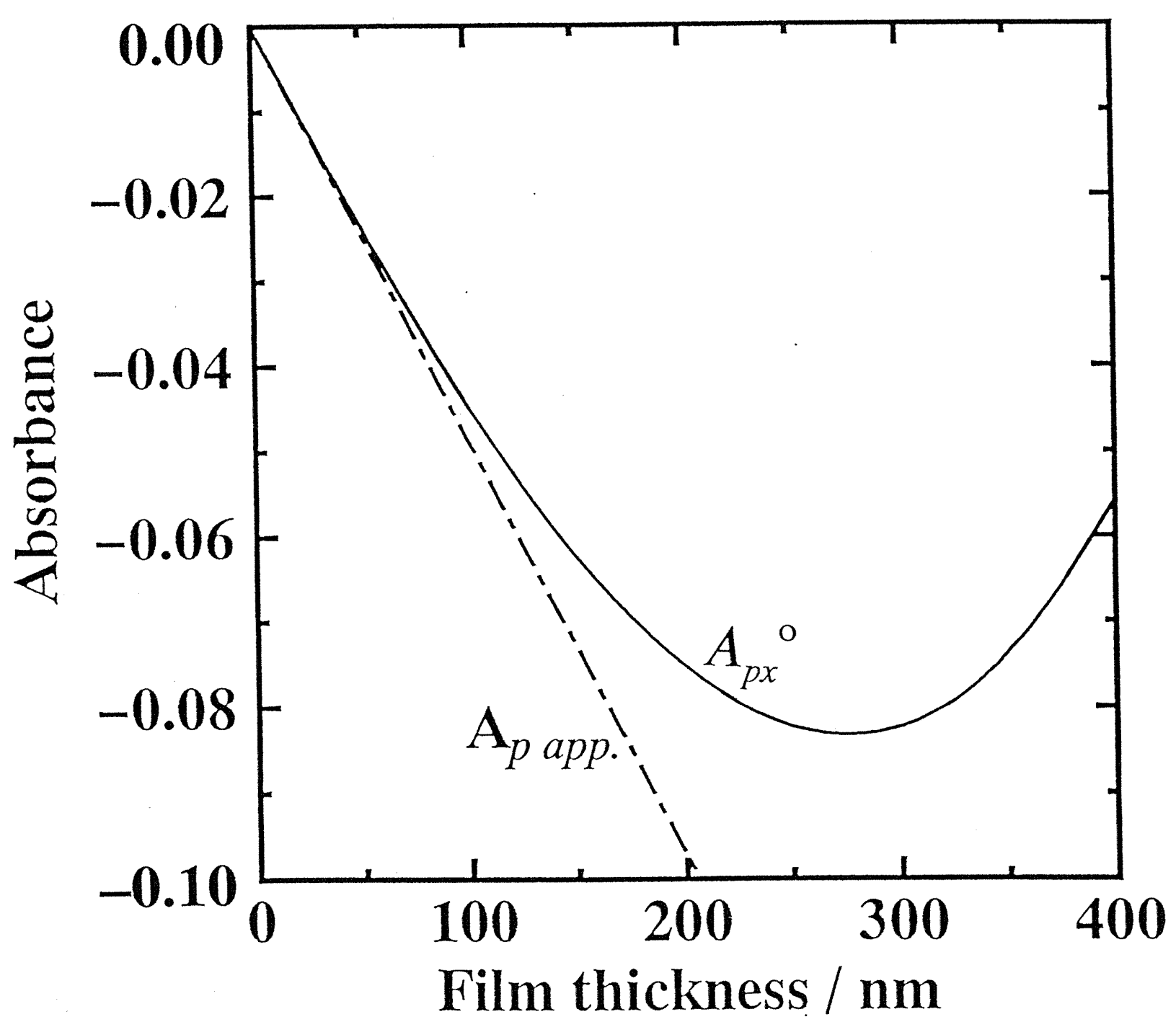

Fig. 5. T. Hasegawa, S. Takeda, A. Kawaguchi and J. Umemura 


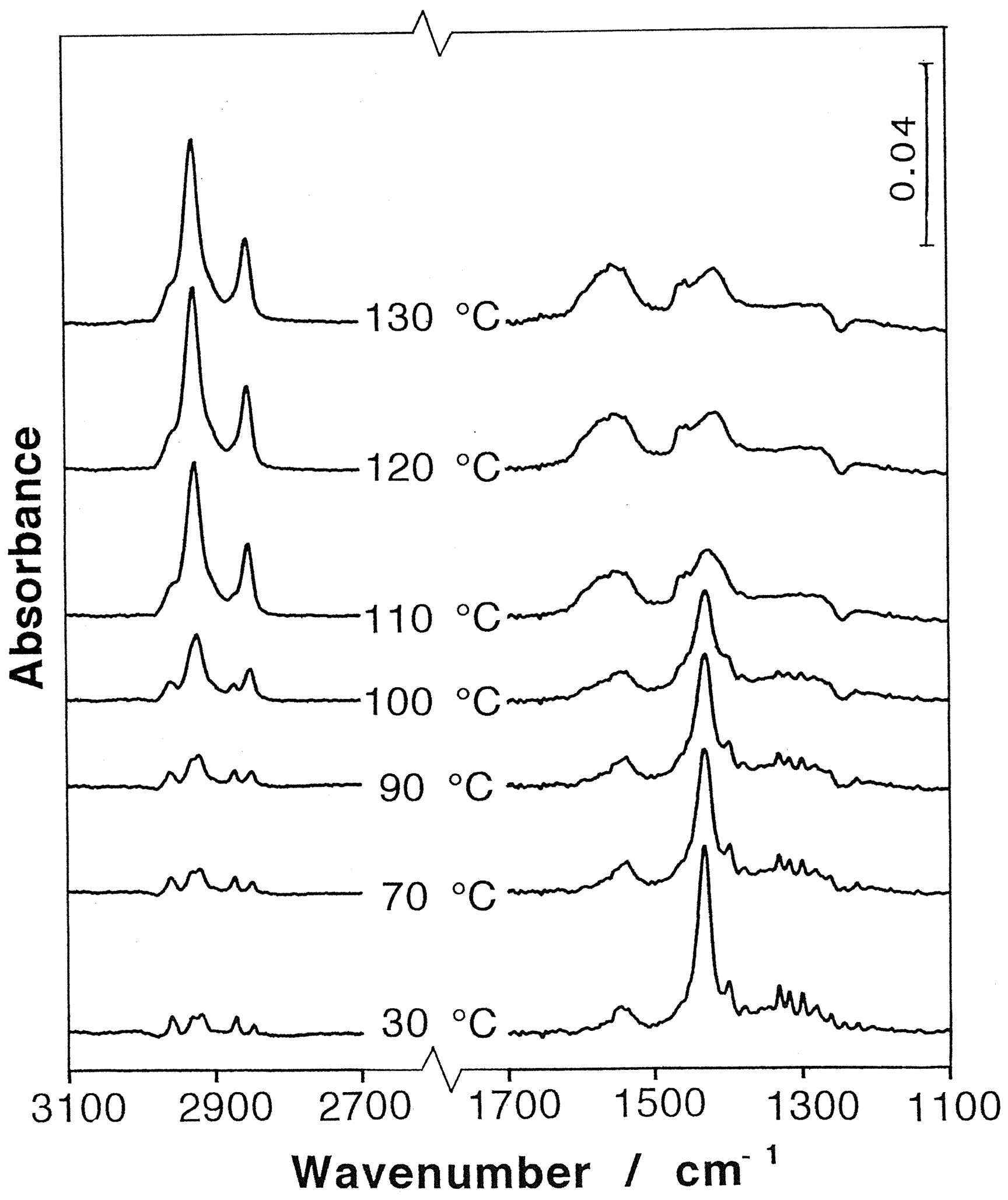

Fig. 6. T. Hasegawa, S. Takeda, A. Kawaguchi and J. Umemura 


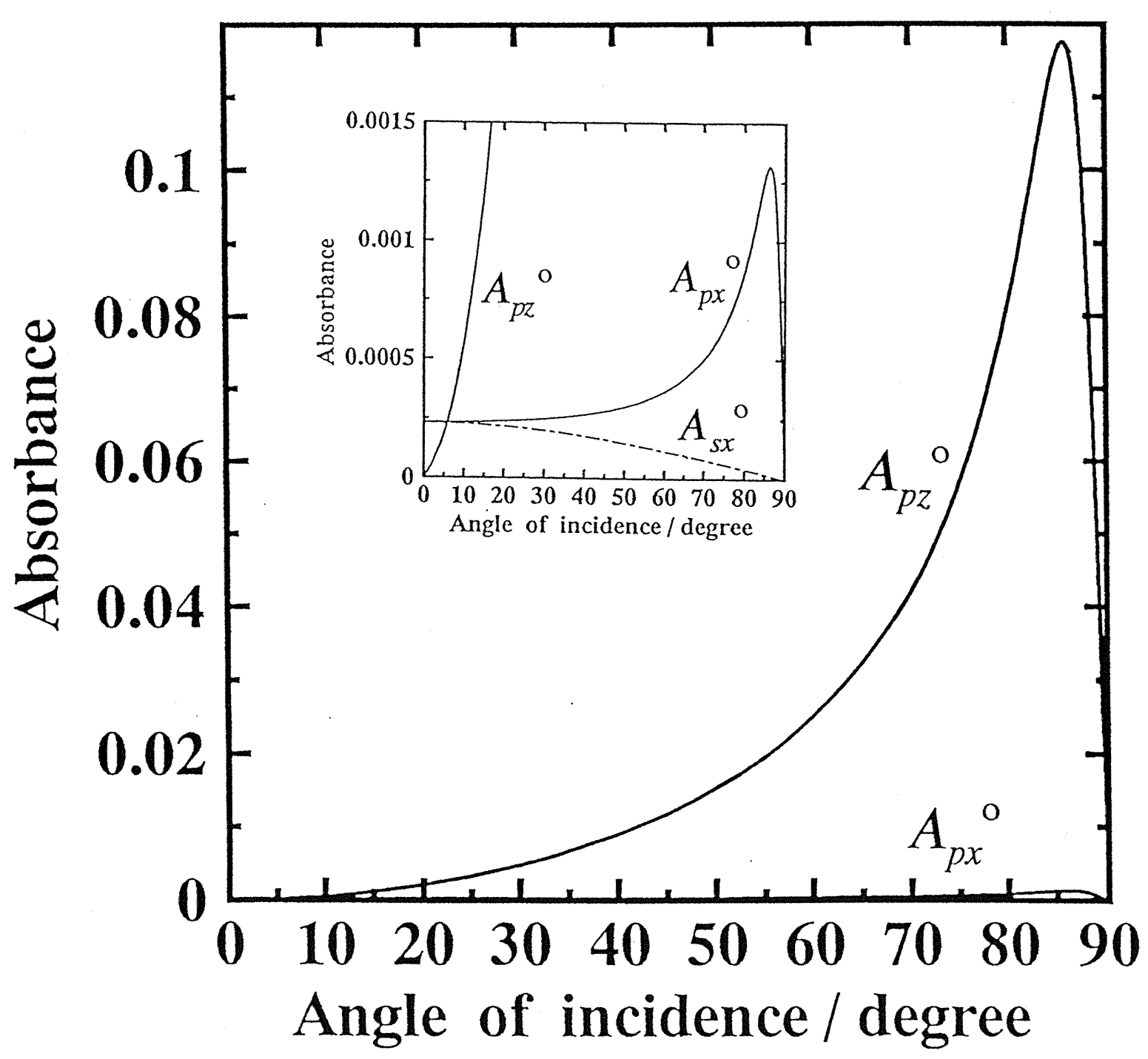

Fig. 7. T. Hasegawa, S. Takeda, A. Kawaguchi and J. Umemura 


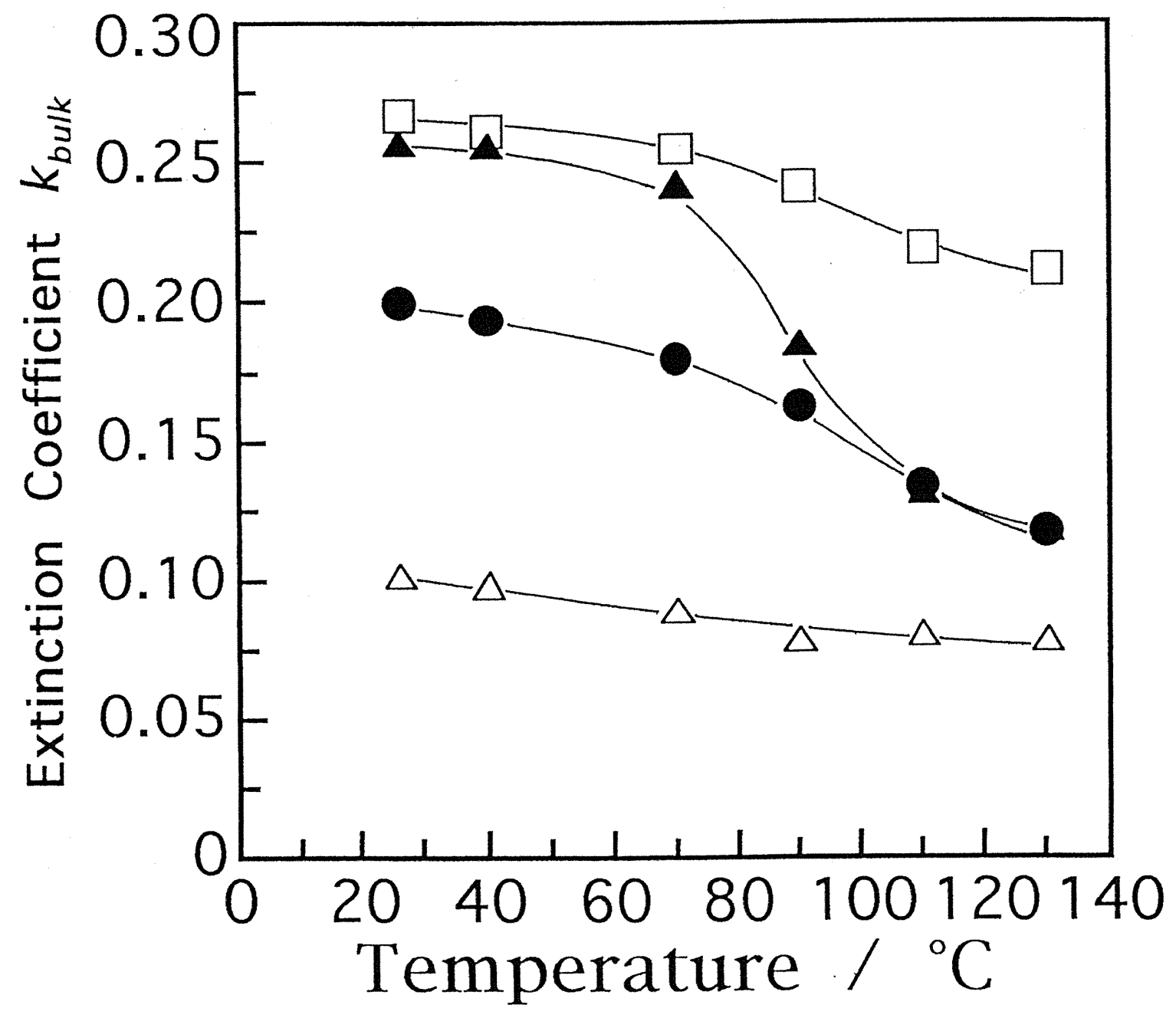

Fig. 8. T. Hasegawa, S. Takeda, A. Kawaguchi and J. Umemura 


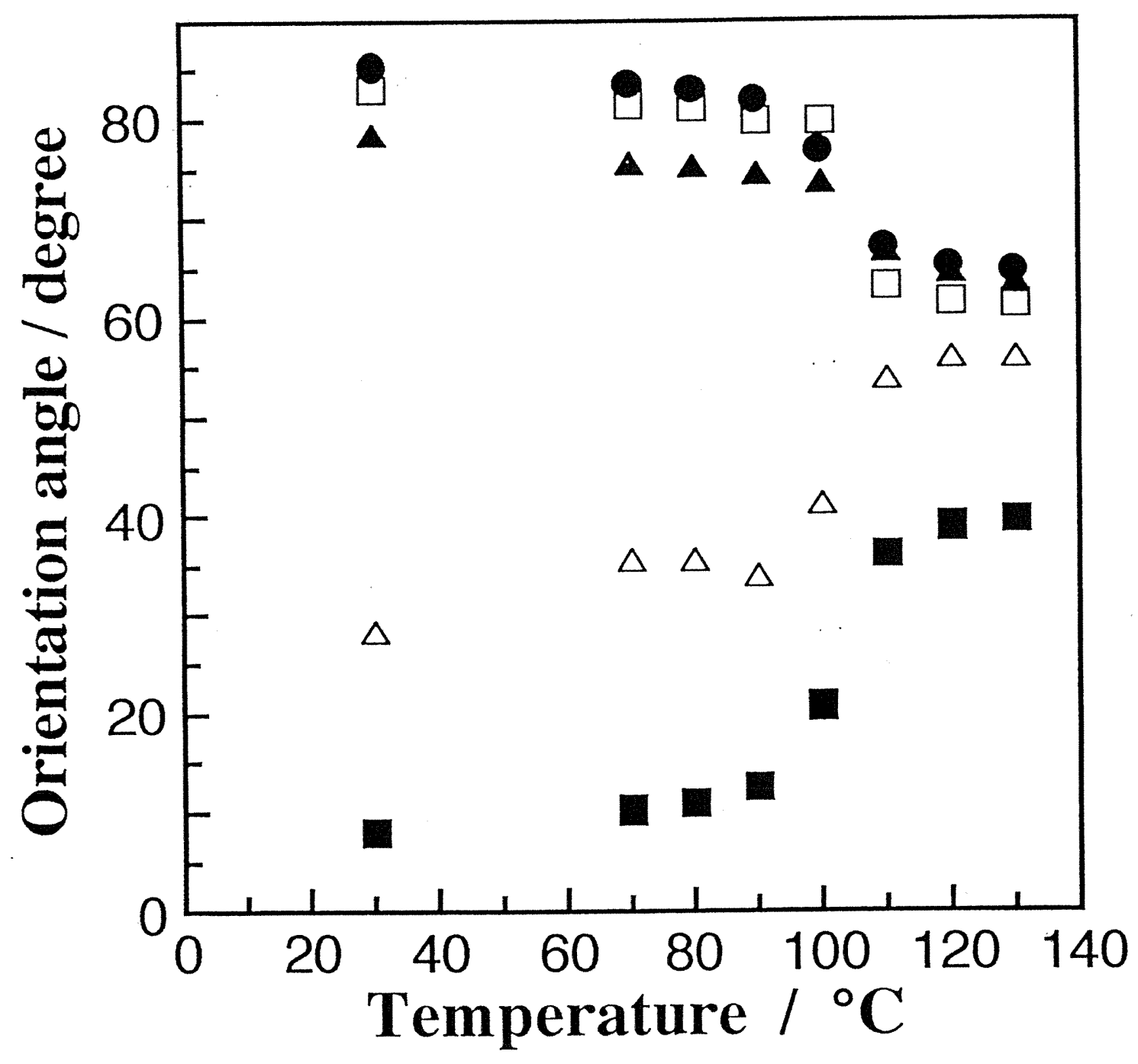

Fig. 9. T. Hasegawa, S. Takeda, A. Kawaguchi and J. Umemura 


\section{8 - cd Stearate}

8 - Cd Stearate- $_{35}$
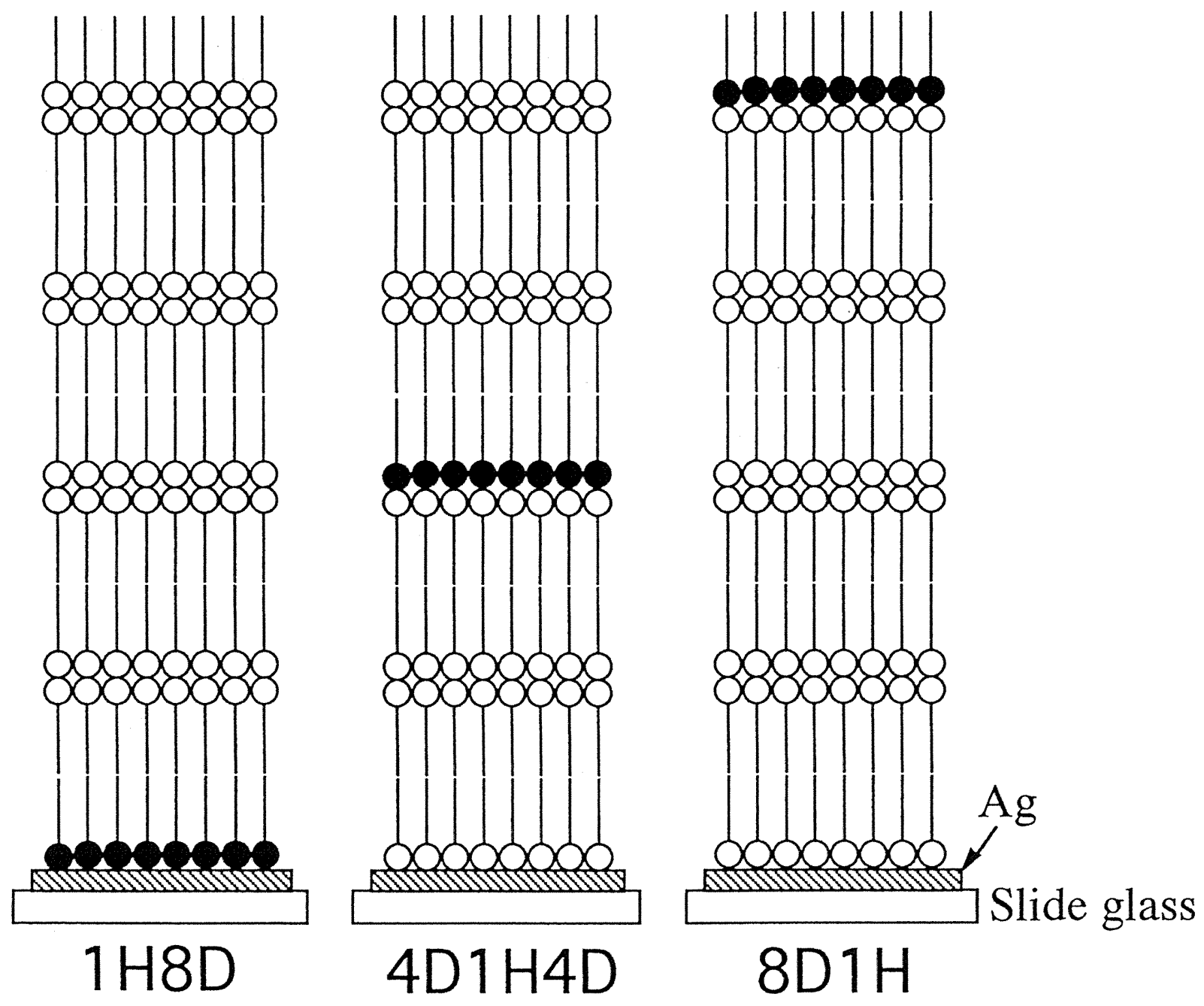

Fig. 10 T. Hasegawa, S. Takeda, A. Kawaguchi and J. Umemura 




Fig. 11. T. Hasegawa, S. Takeda, A. Kawaguchi and J. Umemura 


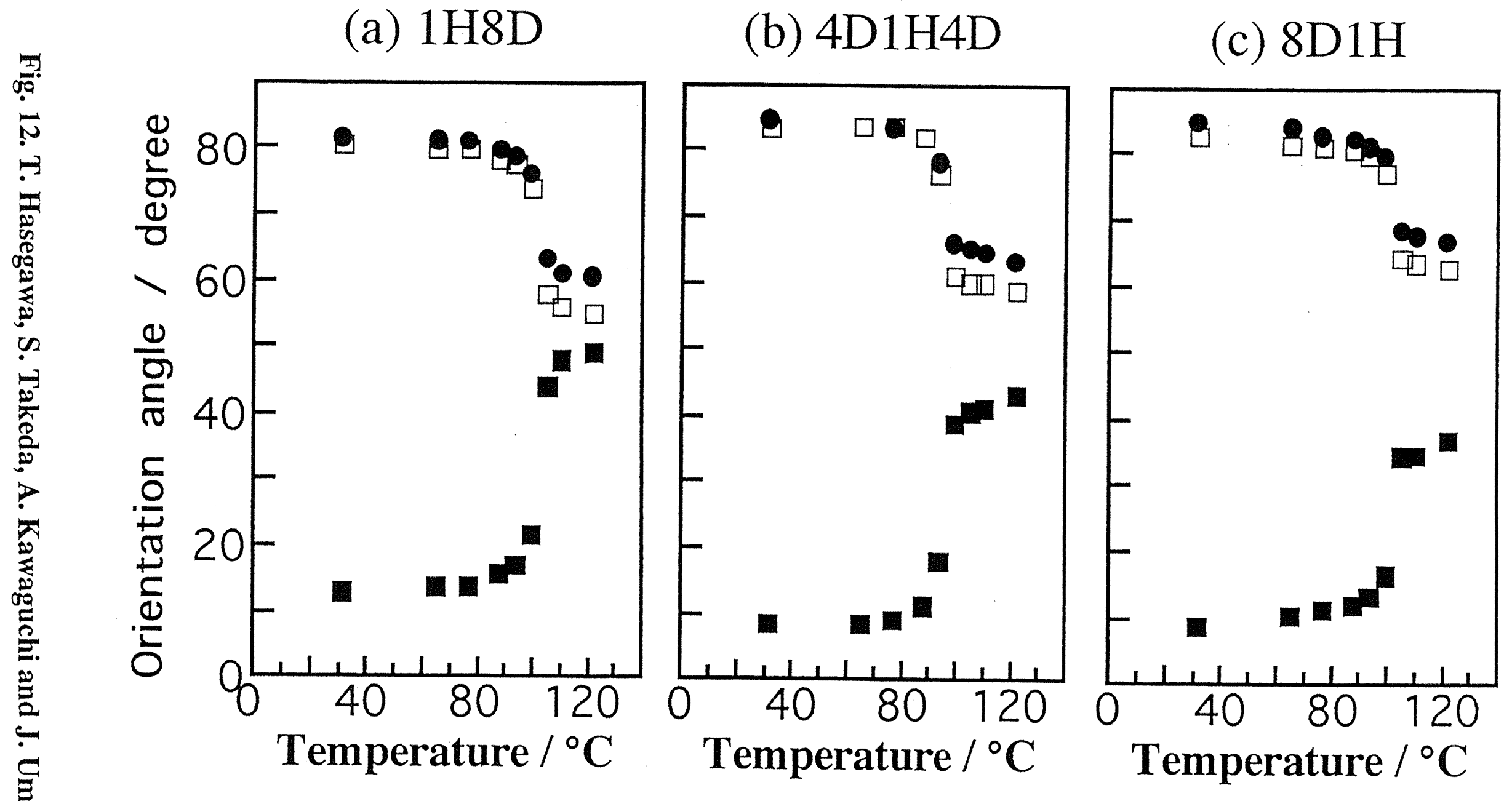

